\title{
Article \\ Facade Style Mixing using Artificial Intelligence for Urban Infill
}

\author{
Ahmed Khairadeen Ali ${ }^{1,2 *}$ and One Jae Lee ${ }^{2}$ \\ 1 Architecture Department, College of Engineering, University of Duhok, Duhok, Iraq: \\ ahmedshingaly@gmail.com \\ 2 Haenglim Architecture and Engineering Company, Republic of Korea; o.lee@haenglim.com \\ Version April 20, 2021 submitted to Journal Not Specified
}

\begin{abstract}
Artificial Intelligence and especially machine learning have noticed rapid advancement on image processing operations. However, its involvement in the architectural design is still in its initial stages compared to other disciplines. Therefore, this paper addresses the issues of developing an integrated bottom up digital design approach and details a research framework for the incorporation of Deep Convolutional Generative Adversarial Network (GAN) for early stage design exploration and generation of intricate and complex alternative facade designs for urban infill. This paper proposes a novel building facade design by merging two neighboring building's architecture style, size, scale, openings, as reference to create a new building design in the same neighborhood for urban infill. This newly produced building contains the outline, style and shape of the parent buildings. A 2D urban infill building design is generated as a picture where 1) neighboring buildings are imported as a reference using mobile phone and 2)iFACADE decode their spatial adjacency. It is depicted the iFACADE will be useful for designers in the early design stage to generate new façades depending on existing buildings in a short time that will save time and energy. Besides, building owners can use iFACADE to show their architects their preferred architecture facade by mixing two building styles and generating a new building. Therefore, it is depicted that iFACADE can become a communication platform in the early design stages between architects and owners. Initial results properly define a heuristic function for generating abstract design facade elements and sufficiently illustrate the desired functionality of our developed prototype.
\end{abstract}

Keywords: : Artificial Intelligence; Generative Adversarial Network; Machine Learning; Computationl Design; Urban infill; Facade design

\section{Introduction}

In general, building envelope design configurations follow similar urban street configurations outlined by city urban policies including building height, width, color, openings, open to built ratio [1]. Therefore, when designing a new building in an existing neighborhood, usually architects follow neighboring buildings architecture style to outline new buildings design mass. In results, Each neighborhood adopts a module that is almost typical and repetitive in all its building massing and the architect's input in designing new buildings minimum due to urban policies and existing neighboring buildings [2].

Conventional building design is an iterative process that is costly and time-consuming. 1) draw bubble diagram of building zones and outline appearances; 2) generate suitable floor plans, elevations and gather input from customers; 3) return to the sophistication bubble diagram, and 4) iterate. Architects and their clients also need to compromise on the quality of the design, given the limited budget and time. In the architecture, design, and real-estate industries, automated building massing generation techniques are therefore in critical demand with immense potential [3]. 
There have recently been several experiments on generative approaches, variational auto-encoders, in the area of computer vision (VAEs) [4] and generative adversarial nets (GANs) [5]. In specific, GANs display important results in different tasks of computer vision, such as image creation [6-8], image conversion [9], super-resolution [10] and text-to-image synthesis [11]. GANs have already been applied to facade generation [8]. proposed Pix2Pix which is based on conditional GAN [5]. By using conditional vectors, we can control the categories or attributes of generated images. Pix2Pix generates facade images with the condition on a masked image that contains predefined labels for each building element. However, both the GANs failed to generate realistic facade images which look realistic.

This paper proposes a novel building facade design by combining the architectural form, height, scale, openings of two adjacent buildings as a guide to construct a modern building design using iFACADE in the same neighborhood for urban infill. The outline, style and type of the parent buildings are used in this newly constructed building. As an image, a 2D urban infill building design is created in which 1) neighboring buildings are imported by cell phone as a reference and 2) iFACADE decodes their spatial adjacency. In the urban neighborhood, a building design is created as an image and enhanced. Major contributions of this paper can be summarized as follows: (1) We propose the style-based conditional generator to control the latent space, (2) Our proposed generator can generate images that reflect each feature from condition information that specifies multiple classes, (3) In the experiments with a facade dataset, we reveal that the noises in the style-based generator indicate additional elements such as building windows, walls, and outlines information, and unseen types of building facade can be generated by mixing multiple kinds of facades with mixing conditional weights.

\section{Literature Review}

\subsection{Generative Adversarial Networks}

Image-to-Image conversion techniques aim to acquire a function of conditional image creation that maps a source domain's input image to a target domain's corresponding image. To solve diverse image-to-image conversion tasks, Isola et al. [12] first proposed the use of conditional GANs. Their thesis has since been expanded to several scenarios: unsupervised learning $[7,8,13]$, multi-domain image synthesis [14], and conditional image synthesis [15]. For their tasks, the above works have built dedicated architectures that include training the generator network. In other hand, our research depends heavily on utilizing conditional styleGAN generator.

Many articles have recently proposed different methods for studying semantic edits of the latent code. A typical technique is to identify linear directions that lead to shifts in a particular binary named attribute, such as human face young to old, or no-smile to smile [6,14]. Abdal et al. [16] learn a translation between vectors in $\mathrm{W}+$, changing a collection of fixed named attributes. Finally, By modifying relevant components of the latent code, Collins et al. [17] performs local semantic editing.

\subsection{Feature Disentanglement}

A few critical problems suffer from the above approaches. The input picture must be invertible, first and foremost, i.e., A latent code that reconstructs the picture needs to exist. The input image domain, however, must usually be the same domain on which the GAN was educated. For several image-to-image activities that convert between two separate data realms, such as segmentation charts, drawings, margins, etc., this constraint is a significant restriction. By optimizing an expanded room, such as $\mathrm{W}+$, this restriction can be bypassed $[16,17]$. In this case, though, the latent space doesn't contain rich semantics for an ambiguous data area. For instance, the latent code would not be semantically relevant even though the input sketches could be inverted into $\mathrm{W}+$ of a StyleGAN trained on faces when translating between sketches and natural face images. Secondly, GAN investment remains overwhelming. In particular, though direct encoding techniques in the restoration standard 
have achieved moderate performance, optimization and hybrid techniques are costly and require considerable time to converge.

\subsection{Facade image generation}

Driven by this realization, this research's end-to-end image-to-image architecture encodes a given actual image directly into the desired latent vector, allowing one to solve the image-to-image challenge directly while enjoying the advantages of a pre-trained StyleGAN without incurring needless limitations.

CycleGAN [7] transforms image to image, but without modifying the image material, this work converts only the style between the two types of images. In the other hand, in this article, by setting several domains as conditions, we exploit the structure by fluctuating transformation and type noise, including two or more types.

\section{Methodology}

This research reviewed current literature in the image to image translation and proposed a model that can generate new building facade by mixing two of existing neighborhood building facades for urban infill. the methodology is divided into two parts. first part discuss the urban infill tool called iFACADE and the second part discuss the development of trained model.

Proposed framework generates an imaginary building from a reference building. Trained with elevations of real buildings, a neural network can transform this into a realistic building. Then, switching between different building elevation references it would be possible to generate different views of the same imaginary building. The iFACADE can produce new and non-existent but realistic images using conditional adversarial neural networks that remember a certain set of features from what it has seen in the past; the same process that we humans undergo when we dream.

\subsection{Urban Infill system architecture}

As every neighborhood has its own regulations for a modular architecture style that all buildings within the neighborhood should follow. Therefore most buildings share the same general building characteristics, colors, window openings, and style. Therefore iFACADE was built to generate a new building elevation depending on existing neighborhood building styles.

The iFACADE framework is divided into four essential stages: a) input reference neighborhood buildings where it needs two reference building facade $2 \mathrm{~d}$ colored images (B1 and B2) as input and neighborhood architecture policies as resource test to help the architect evaluate generated facade correctly; b) Use trained model of iFACADE to input referenced images, extract characteristics and generate a new $2 \mathrm{~d}$ images using style mixing trained model; c) output B0 $2 \mathrm{~d}$ image that contains the characteristics of both referenced images as depicted in Figure 1. d) evaluations where the architect manually evaluates the generated facade and check whether it is compatible with the architecture characteristics of the neighborhood and submit a review report; e) iFACADE team reevaluated trained model and upgraded its system architecture depending on the architect and user's feedback.

The system is built in a cloud and front-back end web site is developed to host trained models. It is critical to have the website to let the users interact easily from anywhere by logging to it. Currently it only has one functionality which is generating style mix images and it could be extended to host more information about each neighborhood's regulations in the future. The user submits a review report and it helps the iFACADE team to evaluate the iFACADe model, upgrade its system architecture and retrain it. Besides, uploaded images by users will be added to the training dataset and expand it which will aid the iFACADE team to generate better and more accurate facades in the future as shown in Figure-1 


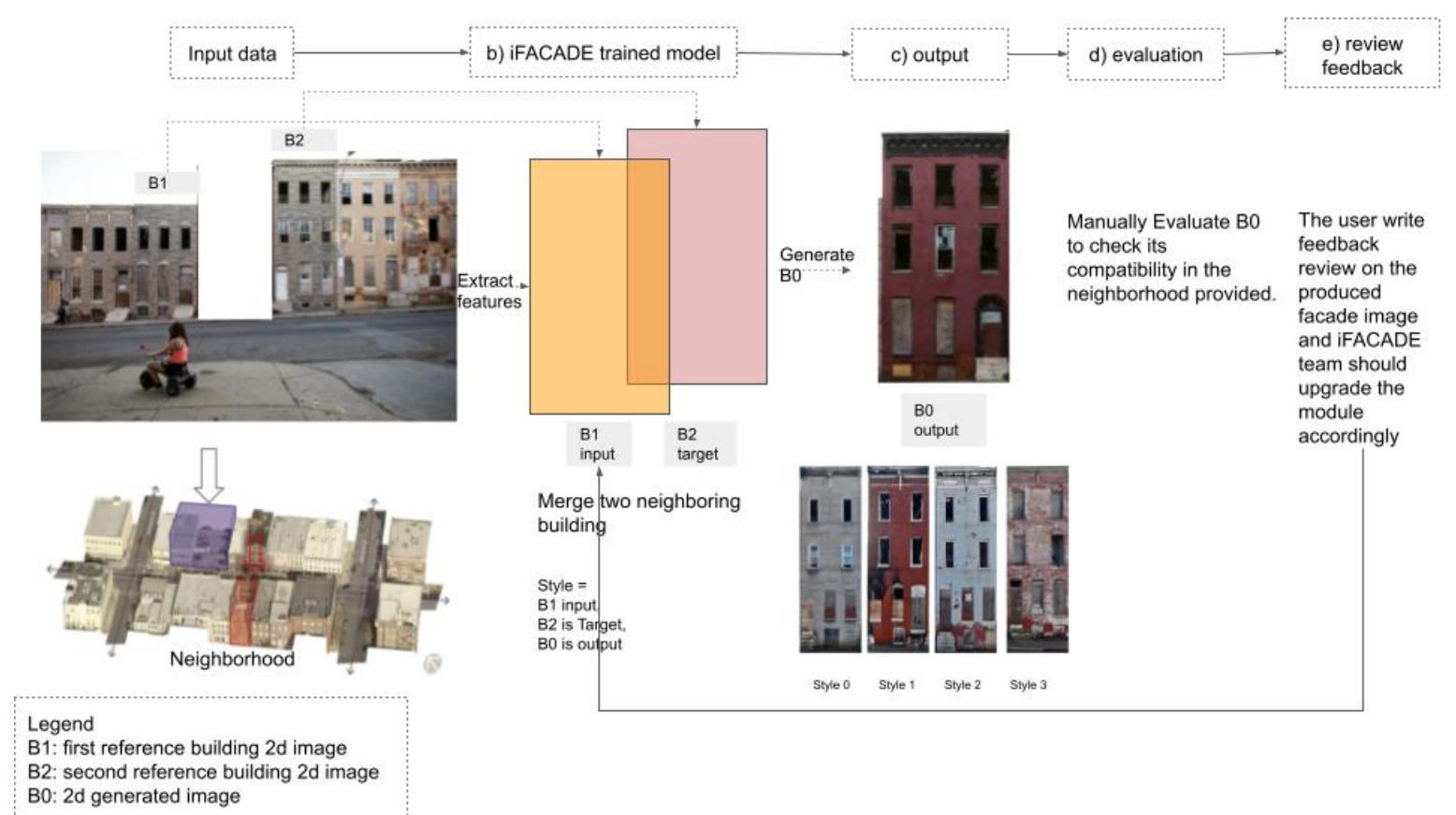

Figure 1. The iFACADE system architecture

\subsection{Style mixing trained model System Architecture}

This subsection explains the iFACADE trained model architecture and methods used to control architecture facade style. Therefore, the main items of the model architecture were explained including the generator, discriminator, style mixing, controlling the latent space, and the dataset used to train the model.

In General, iFACADE model consists of a generator that creates a fake facade image depending on real image input and a discriminator that determines whether the input image is fake or real.

\subsubsection{Conditional style-based generator}

In this subsection, we explain the model generator and custom AdalN architecture. The generator used in iFACADE is a conditional style based neural network that uses transpose convolution to upscale input images. Compared to the traditional stylegan, iFACADE generator controls latent space of conditional stylegan generator by inputting conditional information $(y)$ to the mapping function $(f)$ in the fully connected (FC) layer. This method is adopted from [18].

This research performed a non linear conversion of the latent vector $z$ to conditional style information into two scalers $\left(y_{s}\right.$ and $\left.y_{b}\right)$, where $\mathrm{s}$ stands for scalar and $\mathrm{b}$ stands for bias to control an adaptive instance normalization layer (AdaIN). The AdaIN operation is defined as

$$
\begin{gathered}
y=\left(y_{s}, y_{b}\right)=f(w) \\
\operatorname{AdaIN}\left(x_{i}, y\right)=y_{s} \frac{x_{i}-\mu(x)}{\sigma(x)}+y_{b}
\end{gathered}
$$

Where $x_{i}$ is a normalized instance that we apply AdalN to, $y$ is a set of two scalars $\left(y_{s}, y_{b}\right)$ that control the "style" of the generated image, $f(w)$ represents a learning affine transformation.

In the proposed generator, AdalN operation is normalized to map networks that show various styles. The dimensions of feature map $\mathrm{z}$ is half of the conditional style value. The Objective function used in the stylegan of this research is adopted from [18] that use the hinge version of the standard adversarial loss [6], defined as 

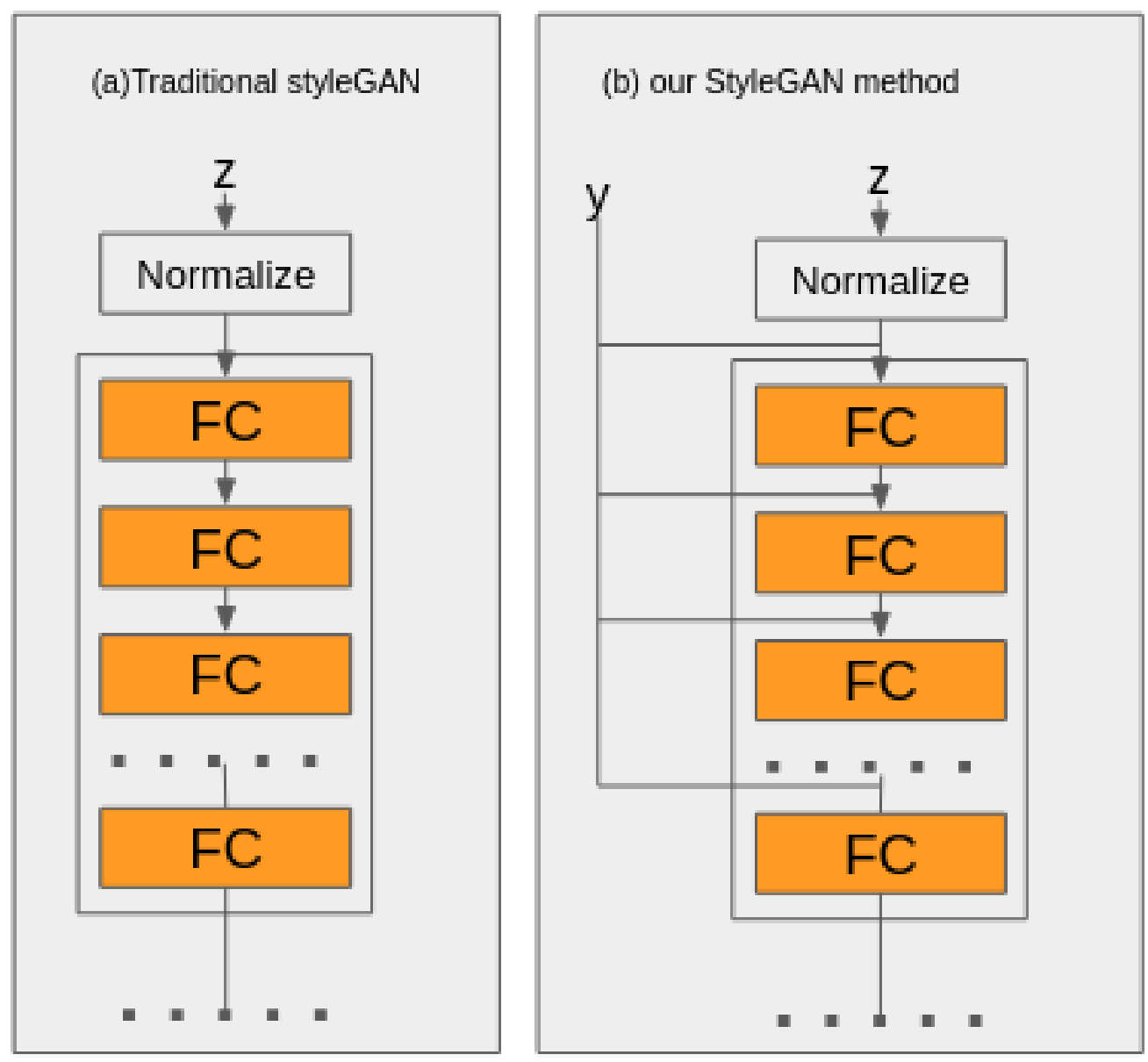

Figure 2. The latent code of a traditional stylegan generator is fed only the input layer $(z)$ on the left. iFACADE added additional conditional information $(y)$ to the styleGAN generator (left). The Conditional code $(y)$ gives more control on the style.

$$
\begin{gathered}
L\left(G^{\wedge}, D\right)=E_{q}(y)\left[E_{q}(x \mid y)[\max (0,1-D(x, y))]\right]+E_{q}(y)\left[E_{p}(z)\left[\max \left(0,1+D\left(G^{\wedge}(z, y), y\right)\right)\right]\right] \\
L\left(G, D^{\wedge}\right)=-E_{q}(y)\left[E_{p}(z)\left[D^{\wedge}(G(z, y), y)\right]\right]
\end{gathered}
$$

where $p(z)$ is the standard Gaussian distribution. The generator's conditional mapping function $\mathrm{z}$ is fed to multiple fully connected (FC) layers. The latent code of a traditional stylegan generator is fed only the input layer $(z)$ on the left. iFACADE added additional conditional information $(y)$ to the styleGAN generator (left). The Conditional code $(y)$ gives more control on the style as depicted in Figure-2.

\subsubsection{Conditional Discriminator}

The discriminator is an unsupervised classifier that determines if the input image is true or fake. The model architecture of discriminator used in iFACADE is adopted by projection discriminator proposed by [6]. Differently from the approach of concatenating the embedded conditional vectors into feature vectors, the projection discriminator integrates conditional vectors. 


\subsubsection{Style transfer}

To further encourage the styles to localize, we employ mixing regularization, where a given percentage of images are generated using two random latent codes instead of one during training. It switches from one latent code to another at a randomly selected point in the synthesis network when producing such an image. To be specific, we run two latent codes $z 1, z 2$ through the mapping network, and have the corresponding $w 1, w 2$ control the styles so that $w 1$ applies before the crossover point and w2 after it. This regularization technique prevents the network from assuming that adjacent styles are correlated.

As a basis for our metric, we use a perceptually-based pairwise image distance that is calculated as a weighted difference between two VGG16 [19] embedding, where the weights are fit so that the metric agrees with human perceptual similarity judgments. If we subdivide a latent space interpolation path into linear segments, we can define the total perceptual length of this segmented path as the sum of perceptual differences over each segment, as reported by the image distance metric. The average perceptual path length in latent space $Z$, over all possible endpoints.

\section{Case Study}

\subsection{Model Training Dataset}

This research trained a conditional generative adversarial network, using customized StyleGAN to reconstruct the facade images from cpm dataset. It uses a total of 720 images of facade that was adopted from CMP, eTRIMS and ECP datasets. CMP dataset contains 606 pairs of annotated and real images of facade images. The dataset is collected by $<>$. The images are from different international cities but they share close modern architecture style with minor detailed architecture style differences that were neglected in this paper. We processed the images manually and chose the best 420 images and erased the rest. The additional images collected from eTRIMS database that contained 60 facade images, and Ecole Centrale Paris facade database. The Facade images collected were processed to 128 * 128 pixels with 3 channels, and divided to 80 percent training, 15 percent test and 5 percent validation. The facade images constraints 12 classes Facade, molding, cornice, pillar, window, door, sill, blind, balcony, shop, deco, and background.

To increase the training speed, the images resolution were decreased to $128^{*} 128$ pixels with three channels, and they are not suitable for high-resolution image generation. This research normalizes the image color values to $[-1,1]$ before feeding the image into the model.

\section{2. iFACADE model training}

This research used Tensorflow to implement the model training. We also start to generate resolutions from $8 \times 8$. The models are optimized by stochastic gradient descent. For all experiments, the learning rate is fixed at 0.002 . it updates the generator once for each discriminator update. We have implemented the proposed architecture in Tensorflow and utilized a workstation with single NVIDIA 2080 Ti GPU. Our model adopts Style-GAN [6] with ADAM optimizer (b1 = 0.5, b2 = 0.999) and is trained for 11 days 6 hours. The learning rates of the generator and the discriminator are 0.0001 , respectively. The batch size is 4 . We set the number of critics to 1 and use leaky-ReLUs $(a=0.1)$ for all non-linearities except for the last one in the generator where we use hyperbolic tangent. styles are configured on a per-image basis through two normalization parameters calculated per feature map, and those parameters are deterministically calculated based on a global style vector $\mathrm{w}$.

\section{Results}

The output has numerous features that are different from the target, and has a somewhat different color, and is especially guessing in the regions where the labels are sparse. But it gets most of the main architectural features in the right place. This leads us to believe that we can mock up whole new label 

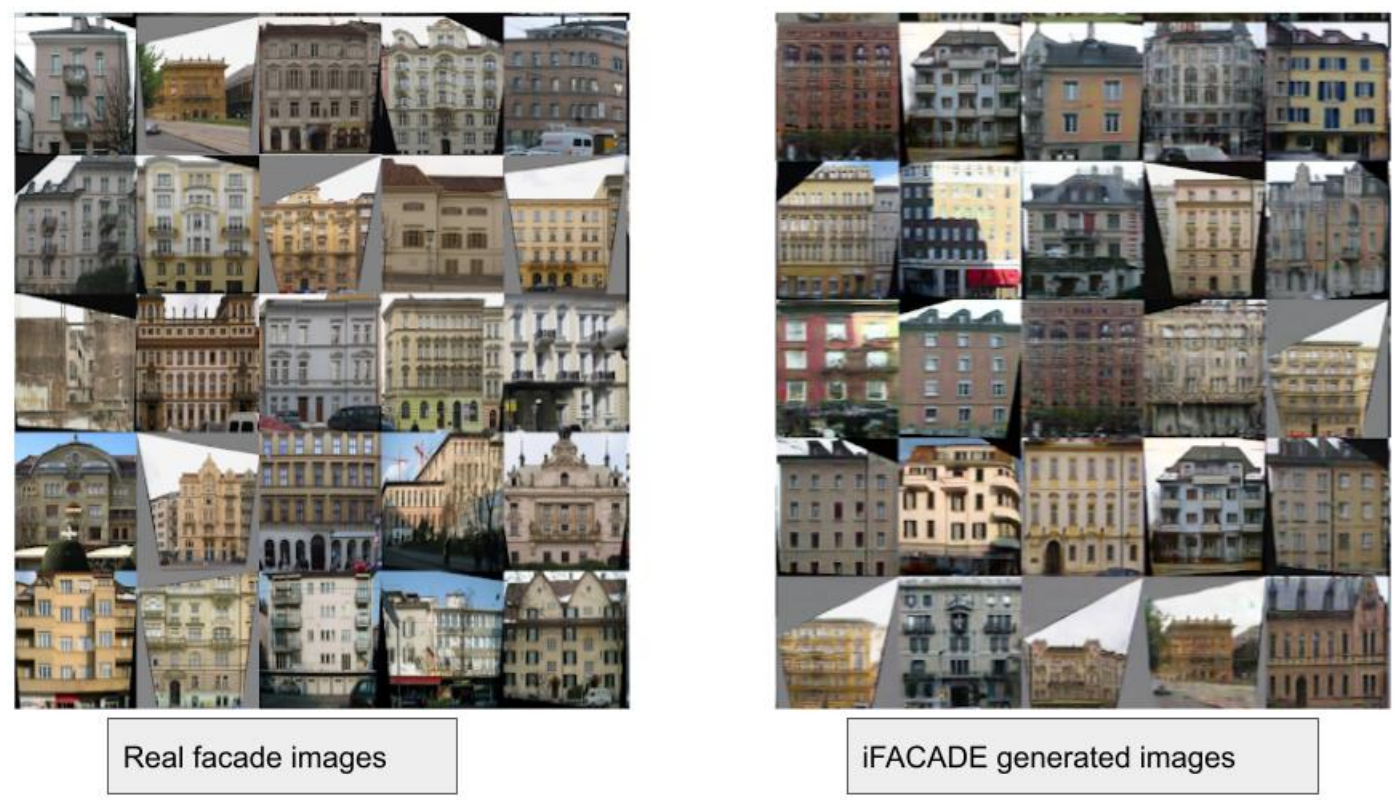

Figure 3. example images generated from inputs containing condition information specifying (a) one, (b) two, (c) three domains, respectively. All images are generated with the same style noise. Our model can generate images that include class information from an input that includes condition information that specifies multiple classes. Style mixing

maps and create realistic looking facades from them! This could be very useful for an architect; they can sketch a design for a building and then quickly prototype textures for it (perhaps choosing from several dozen since they are so easy to produce).

what happens when you combine together feature settings from two different images. Because style injection happens separately at each layer, this can be easily done by just feeding in Building B1's $\mathrm{w}$ vector to some set of layers, and Building B2's w vector to the remaining layers. This results in some layers being configured according to Building B1's parameters, and others configured according to Building B2's. This is what's being shown in the image above: in each row, we're taking the leftmost image and swapping out some group of its style parameters with the image in the corresponding column. For the first three rows, we're swapping in the coarse style parameters from the source, for the second two rows, intermediate, and for the last row, only fine style parameters are being "imported" from the alternate image. (Note that none of these are real images, simply different artificial draws from the input $\mathrm{z}$ distribution, which are then mapped into a $\mathrm{w}$ vector using the mapping network) as shown in Figure-3.

We observe that the style-based generator (E) improves FIDs quite significantly over the traditional generator (B), almost 20 percent, corroborating the large-scale ImageNet measurements made in parallel work [6,5]. Figure 2 shows an uncurated set of novel images generated from the FFHQ dataset using our generator. As confirmed by the FIDs, the average quality is high, and even accessories such as eyeglasses and hats get successfully synthesized. All FIDs in this paper are computed without the truncation trick, and we only use it for illustrative purposes in Figure 2 and the video. All images are generated in 1024 resolution as illustrated in Figure- 4 .

Examples of stochastic variation. (a) Two generated images. (b) Zoom-in with different realizations of input noise. While the overall appearance is almost identical, individual architecture elements are placed very differently. (c) Standard deviation of each pixel over 100 different realizations, highlighting which parts of the images are affected by the noise. training improves the localization considerably, indicated by improved FIDs in scenarios where multiple latents are mixed at test time. 


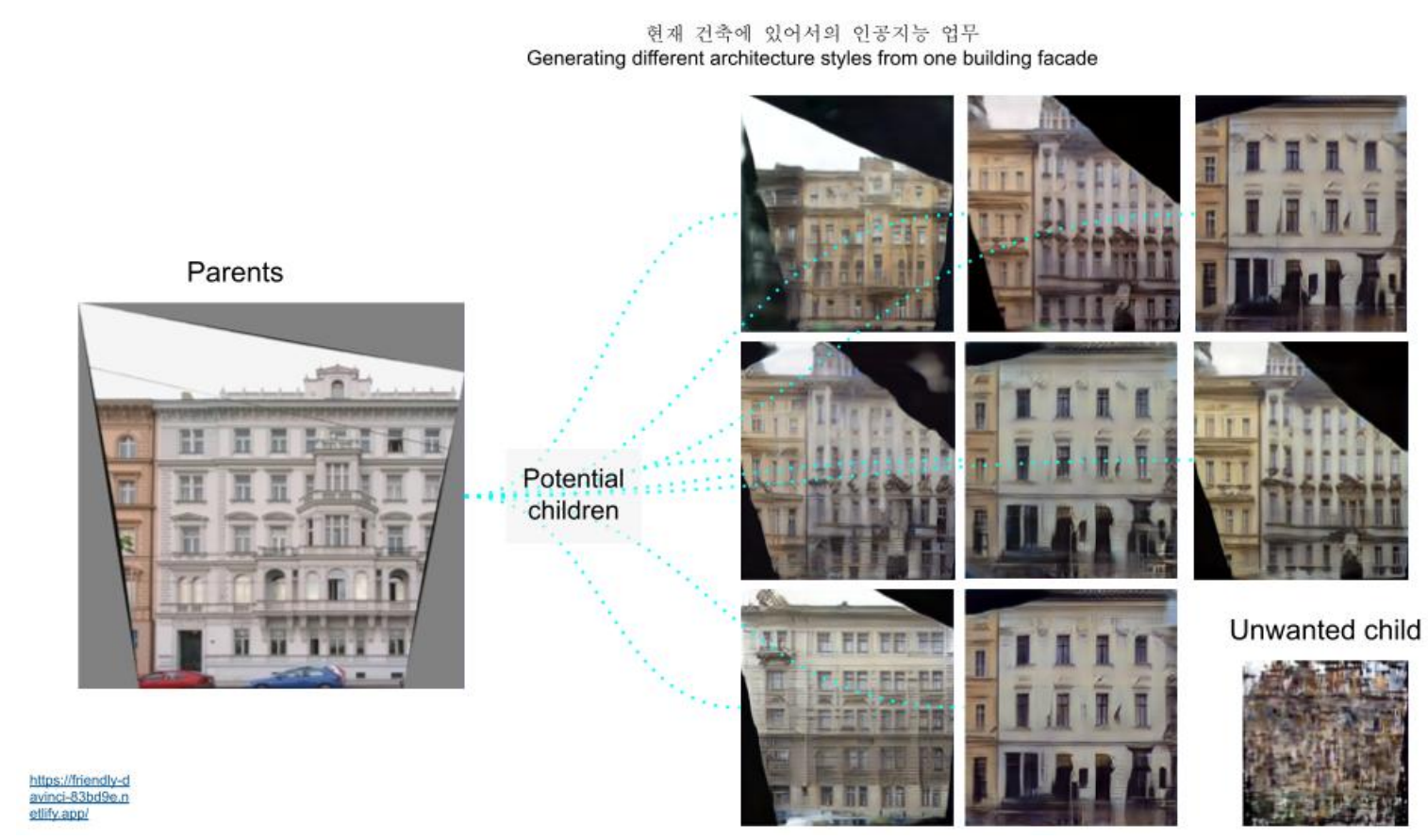

Figure 4. Generating different architecture styles from one building facade by manipulating latent space

Figure 3 presents examples of images synthesized by mixing two latent codes at various scales. We can see that each subset of styles controls meaningful high-level attributes of the image.

\section{Discussion}

It is depicted the iFACADE will be useful for designers in the early design stage to generate new façades depending on existing buildings in a short time that will save time and energy. Besides, building owners can use iFACADE to show their architects their preferred architecture facade by mixing two building styles and generating a new building. Therefore, it is depicted that iFACADE can become a communication platform in the early design stages between architects and owners.

Figure 4 shows that images are generated from fixed the input noises styles, and the condition vectors representing each class by our model. We can see that our model can generate an arbitrary class of images thanks to the condition vectors. However, although the noises and styles are fixed, generated facades have distorted food images because they have few round samples. Figure 5 shows the images generated from a fixed style, a condition vector, and randomly sampled noise. Each image is generated by our model given different random noises. We can see that random noise plays a role in expressing differences such as food topping. Figure- 4 shows that images generated by our model simultaneously with condition vectors representing two or more classes. We can see that our model can generate each feature even when given the multiple condition vectors.

$$
\text { targeted facade }=G(x) \text { variant of target facade }=G(x+\epsilon)
$$

where $\mathrm{G}$ is the generator, and $\epsilon$ is a vector whose components are small numbers that are sampled randomly.

The iFACADE does not work on neighborhoods that do not follow modular architecture styles as generated facades will have characteristics from references building facades. It also cannot apply on new neighborhoods that do not have existing buildings as it depends on prebuilt facade as reference to generate a new building style. Moreover iFACADE cannot control specific characteristics of each building element style;for example, it cannot change the window opening style or porch height 
depending on the user's preferences. That's why, in future iFACADE will have more minor control on each building element depending on the user's preference.

\section{Conclusion}

This research proposes a machine learning tool that can mix facade style of two reference facade images and generate a unique facade design that can be used in the urban infill. Experiments show that the proposed model can generate various facade images with conditional vectors. The proposed tool could be also valuable for facade designers where it's capable of converting an architect's simple building sketch into a prototype texture at a rapid rate. This research's main contributions are: (a) iFACADE can mix facade styles to generate a new facade image that has it's own characteristicsa, (b) the latent space of generated image can be used to control output image style architecture element's style details, (c) Our proposed generator can produce images that represent each function from condition information that defines multiple classes. In the future, iFACADE could be extended to a mobile app that can host trained model where the user can simply take a picture of two desired building elevations and the app will generate mixed style architecture facade. After that, the user can project the generated facade on the unbuilt space using augmented reality technology. Thus, our proposed conditional style-based generator has great potential to solve problems that are still unresolved concerning food images. Finally, we hope that our proposed model will contribute to further architecture design studies. Besides, iFACADE will be extended to generate $3 \mathrm{~d}$ facade elements beside the $2 \mathrm{~d}$ images for it to be used directly in the architecture early design stages directly and increase automation.

Author Contributions: "conceptualization, A.K.; methodology, O.L.; software, A.K.; validation, A.K.; formal analysis, O.L.; investigation, O.L.; resources, O.L.; data curation, A.K.; writing-original draft preparation, A.K.; writing-review and editing, A.K.; visualization, A.K.; supervision, O.L.; project administration, O.L.; funding acquisition, O.L."

Acknowledgments: This work is supported by Haenglim Architecture and Engineering Company. The first author would like to thank Chung Ang University and the Korean Government Scholarship program.

Conflicts of Interest: "The authors declare no conflict of interest."

\section{References}

1. Adamus-Matuszyńska, A.; Michnik, J.; Polok, G. A Systemic Approach to City Image Building. The Case of Katowice City. Sustainability 2019, 11, 4470.

2. Talen, E. City rules: How regulations affect urban form; Island Press, 2012.

3. Touloupaki, E.; Theodosiou, T. Performance simulation integrated in parametric 3D modeling as a method for early stage design optimization-A review. Energies 2017, 10, 637.

4. García-Ordás, M.T.; Benítez-Andrades, J.A.; García-Rodríguez, I.; Benavides, C.; Alaiz-Moretón, H. Detecting Respiratory Pathologies Using Convolutional Neural Networks and Variational Autoencoders for Unbalancing Data. Sensors 2020, 20, 1214.

5. Goodfellow, I.; Pouget-Abadie, J.; Mirza, M.; Xu, B.; Warde-Farley, D.; Ozair, S.; Courville, A.; Bengio, Y. Generative adversarial networks. Communications of the ACM 2020, 63, 139-144.

6. Karras, T.; Laine, S.; Aittala, M.; Hellsten, J.; Lehtinen, J.; Aila, T. Analyzing and improving the image quality of stylegan. Proceedings of the IEEE/CVF Conference on Computer Vision and Pattern Recognition, 2020, pp. 8110-8119.

7. Almahairi, A.; Rajeswar, S.; Sordoni, A.; Bachman, P.; Courville, A. Augmented cyclegan: Learning many-to-many mappings from unpaired data. arXiv preprint arXiv:1802.10151 2018.

8. Zhu, J.Y.; Zhang, R.; Pathak, D.; Darrell, T.; Efros, A.A.; Wang, O.; Shechtman, E. Toward multimodal image-to-image translation. Advances in neural information processing systems, 2017, pp. 465-476.

9. Zhang, Y.; Yin, Y.; Zimmermann, R.; Wang, G.; Varadarajan, J.; Ng, S.K. An Enhanced GAN Model for Automatic Satellite-to-Map Image Conversion. IEEE Access 2020, 8, 176704-176716.

10. Bulat, A.; Yang, J.; Tzimiropoulos, G. To learn image super-resolution, use a gan to learn how to do image degradation first. Proceedings of the European conference on computer vision (ECCV), 2018, pp. 185-200. 
11. Reed, S.; Akata, Z.; Yan, X.; Logeswaran, L.; Schiele, B.; Lee, H. Generative adversarial text to image synthesis. arXiv preprint arXiv:1605.05396 2016.

12. Isola, P.; Zhu, J.Y.; Zhou, T.; Efros, A.A. Image-to-image translation with conditional adversarial networks. Proceedings of the IEEE conference on computer vision and pattern recognition, 2017, pp. 1125-1134.

13. Zhu, J.Y.; Park, T.; Isola, P.; Efros, A.A. Unpaired image-to-image translation using cycle-consistent adversarial networks. Proceedings of the IEEE international conference on computer vision, 2017, pp. 2223-2232.

14. Choi, Y.; Choi, M.; Kim, M.; Ha, J.W.; Kim, S.; Choo, J. Stargan: Unified generative adversarial networks for multi-domain image-to-image translation. Proceedings of the IEEE conference on computer vision and pattern recognition, 2018, pp. 8789-8797.

15. Mao, Q.; Lee, H.Y.; Tseng, H.Y.; Ma, S.; Yang, M.H. Mode seeking generative adversarial networks for diverse image synthesis. Proceedings of the IEEE Conference on Computer Vision and Pattern Recognition, 2019, pp. 1429-1437.

16. Abdal, R.; Qin, Y.; Wonka, P. Image2stylegan: How to embed images into the stylegan latent space? Proceedings of the IEEE international conference on computer vision, 2019, pp. 4432-4441.

17. Collins, E.; Bala, R.; Price, B.; Susstrunk, S. Editing in Style: Uncovering the Local Semantics of GANs. Proceedings of the IEEE/CVF Conference on Computer Vision and Pattern Recognition, 2020, pp. 5771-5780.

18. Horita, D.; Shimoda, W.; Yanai, K. Unseen food creation by mixing existing food images with conditional stylegan. Proceedings of the 5th International Workshop on Multimedia Assisted Dietary Management, 2019, pp. 19-24.

19. Han, S.; Mao, H.; Dally, W.J. Deep compression: Compressing deep neural networks with pruning, trained quantization and huffman coding. arXiv preprint arXiv:1510.00149 2015. 